\title{
Superfluidity and Pairing Phenomena from Cold Atomic Gases to Neutron Stars
}

\author{
Armen Sedrakian' ${ }^{1}$. J. W. Clark ${ }^{3,4}$. \\ Eckhard Krotscheck ${ }^{2}$
}

Published online: 12 October 2017

(C) Springer Science+Business Media, LLC 2017

Sixty years ago, the proof of the Cooper theorem provided the key to understanding pairing phenomenon in interacting many-fermion systems, triggering creation of the BCS microscopic theory of electronic superconductivity. Since that landmark development, the basic paradigm of BCS theory has been extended to diverse fermionic systems, with considerable success. Exploration of its broader application, modification, and implementation is currently as lively as never before. Indeed, this last decade has witnessed remarkable progress in our understanding of fermionic pairing across an array of systems characterized by vastly different energy scales. In parallel with the ongoing effort to uncover the mechanism responsible for high-temperature electron superconductivity in solids as studied in traditional condensed-matter laboratories, there has been intense activity in the exploration of pairing phenomena in novel systems such as magnetically trapped ultra-cold atomic vapors and in complex many-body systems including liquid ${ }^{3} \mathrm{He}$, finite nuclei, nucleonic matter existing in neuron stars, and quantum chromodynamics.

Since pairing phenomena are a common theme in quantum fluids, condensed-matter systems, nuclear physics, and astrophysics, the range of subjects covered in this volume of the Journal of Low Temperature Physics is broader than the usual scope of this journal. They address predominanly two relates issues:

\footnotetext{
$\bowtie$ Eckhard Krotscheck

eckhardk@buffalo.edu

1 Frankfurt Institute for Advanced Studies, 60438 Frankfurt, Germany

2 Department of Physics, University at Buffalo SUNY, Buffalo, NY 14260, USA

3 Department of Physics and McDonnell Center for the Space Sciences, Washington University, St. Louis, MO 63130, USA

4 Centro de Investigação em Matemática e Aplicações, University of Madeira, 9020-105 Funchal, Madeira, Portugal
} 
- From the methodological perspective, we cover progress toward the development of realistic and reliable microscopic models of pair-correlated fermionic systems. The strengths and weaknesses of various ab initio computational approaches to pairing problems are discussed. These approaches include, on the one hand, semianalytic methods involving (i) continuum Green's functions evaluated through physically motivated approximation schemes and (ii) correlated basis functions together with Fermi hypernetted-chain cluster resummations; and on the other hand, numerically oriented treatments including (iii) Monte-Carlo-based wavefunction approaches and (iv) field-theoretic methods implemented on a lattice. In differing ways, all of these approaches aim toward quantitative determination of corrections of self-energies and effective interactions beyond mean-field approximations. An important aspect is the quantitative role of fundamental three-body forces which is addressed in one of the papers.

- The second focus of this volume is on conceptually novel aspects of pairing expressed in non-BCS situations; these include asymmetrical pairing, phaseseparated pairing states, Cooper pairs with nonzero total momentum, gapless superconductivity. The status of microscopic theoretical description of these aspects in concrete physical examples will be discussed, along with the results for systems that feature traditional pairing.

The repertoire of methods for quantitative microscopic description of quantum many-body systems covers a wide range, as indicated above. In application to concrete physical systems rather than toy models, each of these approaches may require feedback from some alternative method, so as to enhance either accuracy or intuitive access to the physical processes in play. For example, numerical ab initio simulation in configuration space is capable of high precision, whereas application of a semianalytic Green's function approach provides a better control and apprehension of the underlying physics, despite limited accuracy due to some necessary approximation. Such considerations are especially important for strongly correlated systems and at high densities as encountered in dense neutron-star matter-emphatically so when pairing is involved, given that the BCS pairing gap is exponentially sensitive to inputs for the pairing interaction and self-energies. Optimistically, confrontation of the results produced by the existing calculational methods will stimulate the emergence of hybrid theoretical approaches that lead to a deeper and more comprehensive understanding of pair-correlated systems across the broad range of energy scales represented.

There exists a tension between factors that favor standard Cooper pairing due to an attractive force between fermions, and factors that disfavor it, as when conservation laws separate the Fermi momenta of different fermion species. Other complications arise from the state dependence and momentum-space structure of the realistic fermion-fermion interactions, from medium-modification of these interactions and fermion self-energies, and from retardation effects due to finite propagation times of exchange bosons. To understand the implications of such conflicts and complications, there is the need to extend the standard BCS theory to accomodate a much wider class of possible fermionic pairing patterns. The topic of unconventional fermionic pairing is particularly rich in opportunities for interdisciplinary cross-fertilization, because it draws together theoretical ideas from atomic physics, condensed-matter physics, 
nuclear physics, particle physics, and astrophysics. These ideas have direct relevance to experiment and observation. Pairing in isospin-asymmetric nuclear systems is an important facet of the physics of nuclei far from stability being created at radioactive ion-beam facilities and in natural stellar nucleosynthesis, as well as the physics of neutron-star interiors, not to mention QCD at finite density. Experimental studies of ultra-cold, trapped atomic gases continue to energize one of the most successful and fast-growing areas of mainstream physics, situated at the crossroads of new thrusts in condensed-matter physics, atomic physics, quantum optics, QCD, and quantuminformation science.

Unfortunately, one of the contributions to this collection of papers has been published erroneously in the November issue of the same volume of JLTP, it addresses the issue of $\alpha$-clustering in nuclear matter. The interested reader is directed to the publication "Composition of Nuclear Matter with Light Clusters and Bose-Einstein Condensation of $\alpha$ Particles” by X.-H. Wu, S.-B. Wang, A. Sedrakian, and G. Röpke Volume 189, Page 133 (2017) (doi:10.1007/s10909-017-1795-x).

Acknowledgements We thank the European Center for Theoretical Physics in Nuclear Physics and Related Areas $\left(\mathrm{ECT}^{*}\right)$ in Trento for supporting a workshop on the cross-disciplinary pairing phenomena that are the subjects of this Special Volume of JLTP. 